

\title{
Cambio in statu quo ante
}

\author{
Ing. Minor Murillo Chacón. \\ Escuela de Ingeniería Civil \\ Universidad Fidélitas \\ Costa Rica \\ minor.murillo.chacon@gmail.com
}

\begin{abstract}
It is common for young people who decide to venture into the Civil Engineering career have as their main objective "to win the course", studying specifically to pass the subjects of the curriculum.

For more than a decade, the author has implemented a learning methodology focused on the professional growth of the individual facing real situations that are part of the professional exercise that promotes the research and analysis of each of them.Additionally, this document considers the assessment of several engineers in training who have had the opportunity to participate in this type of learning.
\end{abstract}

Keywords: Professional growth, Learning, Junior Engineer.

\section{Introducción}

Ladocencia en las escuelas de Ingeniería Civil se fundamenta principalmente en un proceso de enseñanza-aprendizaje tradicional a cargo de ingenieros experimentados que facilitan los conceptos teóricos y comparten experiencias que contribuyen a la formación de la próxima generación de profesionales.

En pocas ocasiones se cuenta con profesionales en Ingeniería Civil que hayan tenido una formación docente por lo que el proceso pedagógico se basa en un programa establecido y monitoreado por la Escuela.

Sin embargo, hay otras formas de enseñanza que cambian la experiencia del estudiante durante su proceso de formación que le permiten ir creciendo profesionalmente y literalmente "enamorarse de la carrera".

Aplicando el método científico (observando, preguntando, experimentando, analizando y concluyendo) el autor ha implementado una forma diferente de enseñanza donde los participantes descubren el conocimiento al compartir experiencias propias del ejercicio profesional.
Parte del cambio consiste en dejar de considerar a los participantes del curso como estudiantes y empezar a interactuar con ellos como "Ingenieros en formación".

\section{Propuesta actual de la Escuela de Ingeniería Civil}

Los objetivos que mueven el quehacer de la Escuela de Ingeniería Civil de la Universidad Fidélitas de Costa Rica, buscan convertir esta casa de estudios en la universidad referente en Ingeniería Civil.

Actualmente existe una preocupación por contar con las herramientas tecnológicas más modernas que sirvan de apoyo en la formación del estudiante.

El objetivo de la Escuela es la de "formar profesionales capaces de analizar, diseñar y construir obras orientadas al uso civil de la población".

Alineado con esta visión, es posible crear una experiencia de crecimiento profesional donde cada lección impartida en cada uno de los cursos se convierte en una excelente oportunidad para presentar la "materia" como una nueva herramienta para el ejercicio profesional y cada tema se vuelve en una nueva adquisición para la caja de herramientas del ingeniero en formación.

\section{Experiencias de referencia}

El Centro Universitario Moacyr Sreder Bastos (UNIMSB) de Río de Janeiro, tiene la modalidad de internado supervisado donde se le permite al estudiante experimentar lo que se está aprendiendo en la Facultad, recibiendo clases en proyectos, oficinas de consultoría, instituciones del estado; todo esto bajo la tutela de un ingeniero experimentado que funge como docente.

Otro caso muy conocido en nuestro país es la forma de la enseñanza en la Escuela de Medicina en la mayoría de las universidades, ya sea la estatal o las privadas, donde los 
estudiantes realizan la pre-carrera en el campus y luego se trasladan a los diferentes hospitales donde reciben clases en las aulas y auditorios del centro médico y luego salen en pequeños grupos liderados por un Médico experimentado hacia las diferentes áreas o departamentos para aplicar el conocimiento recién adquirido, bajo la modalidad de ejercicio profesional supervisado.

\section{Metodología}

El primer cambio que tuve que realizar fue mental y consistió en dejar de ver estudiantes sentados en el aula esperando una disertación magistral de materia junto con la resolución de problemas, para verlos como ingenieros en formación con deseos de desarrollarse y enamorarse de la carrera. O sea, ;tratarlos como ingenieros!

El ejercicio de aprendizaje se complementó con la aplicación de documentos de uso diario para los Ingenieros Civiles y no en un libro de texto. Específicamente códigos y guías del Instituto Americano del Concreto (ACI por sus siglas en inglés), Especificaciones y normas de la Asociación Americana de Ensayo de Materiales (ASTM por sus siglas en inglés) y el manual para el diseño y control de mezclas de concreto de la Asociación del Cemento Portland (PCA por sus siglas en inglés), entre otros.

El reto aquí es la motivación para que estos nuevos ingenieros "lean los documentos" ya que la mayoría de los ingenieros no somos muy dados a la lectura. Estos documentos son de consulta y guía para Ingenieros Diseñadores, Inspectores y Constructores, publicado por reconocidas instituciones internacionales.

Una vez que varios miembros del grupo empiezan a crecer en su conocimiento a través de los documentos, convencen al resto que la lectura es agradable y sobre todo que se aprende.

Adicionalmente, se organizan en grupos de tres o cuatro integrantes (dependiendo de la matrícula) para formar empresas donde ellos son los directores y asumen responsabilidades gerenciales. Cada empresa debe entregar al docente lo siguiente:

- Visión

- Misión

- Localización de la empresa

- Portafolio de productos

- Organigrama

- Cuadro con los salarios de todos los colaboradores de la empresa con las respectivas cargas sociales.

- Layout de la planta de producción para los casos en que aplica.

Cada empresa tiene la potestad de contratar a nuevos integrantes (observan un buen elemento en otra de las empresas y desean integrarlo al equipo de trabajo) ofreciéndole un mejor salario o bien, prescindir de algún miembro que no cumpla con las expectativas y responsabilidades del grupo, quien es despedido formalmente de la empresa. Todos estos movimientos van asociados a cambios en el cuadro de costos y salarios de cada empresa involucrada.

Para afirmar el conocimiento obtenido por la lectura de los documentos, temas discutidos en clase o la compresión adquirida en las visitas técnicas; cada grupo debe buscar una obra en construcción que se encuentre en desarrollo con el fin de visitarla cada vez que se concluye un tema y revisar si se aplican los conceptos aprendidos. Al final del curso, cada empresa entrega un informe técnico con los hallazgos y oportunidades de mejora, fundamentados en la documentación y conocimiento acumulado durante todo el curso.

También, cada empresa participa en una licitación la cual es tomada del Sistema Integrado de Compras Públicas (SICOP), la cual es analizada y punteada de acuerdo a los diferentes componentes de la oferta: plazo de entrega, memoria de cálculo, certificados de calidad, diseño del elemento, precio, entre otros. O sea, no solo el precio es determinante. Este ejercicio corresponde a la evaluación de un examen parcial y requiere de un trabajo arduo del grupo.

Se han coordinado seis visitas técnicas donde el ingeniero en formación tiene contacto con la realidad del negocio, comprende los procesos y normativa que involucran el desarrollo de determinada actividad y lo más importante, empiezan a tener "contactos" con personal técnico y profesional que serán de mucha utilidad en sus primeras experiencias en el ejercicio profesional.

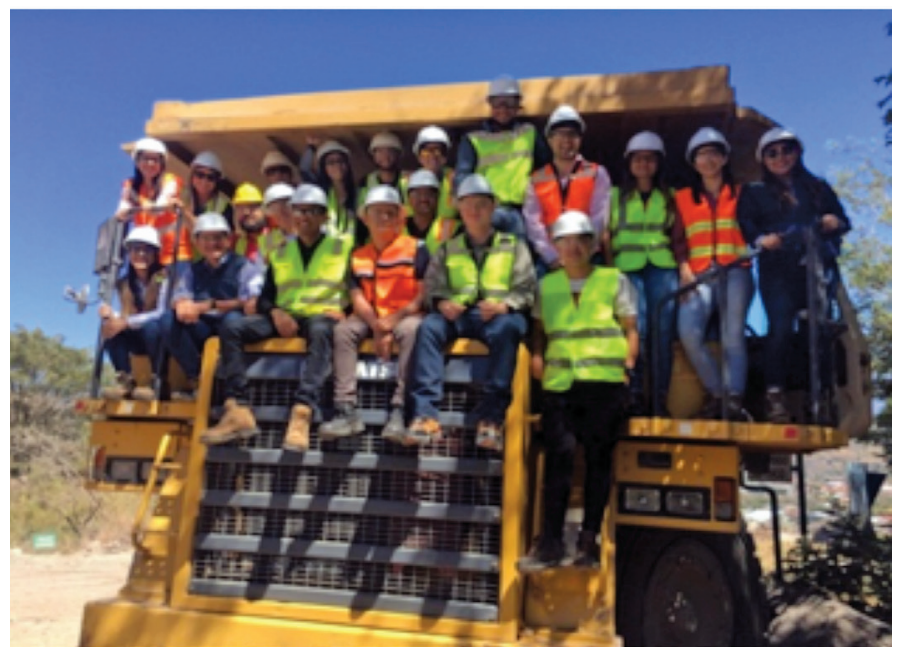

Fig. 1: Visita técnica a una fuente de agregados 


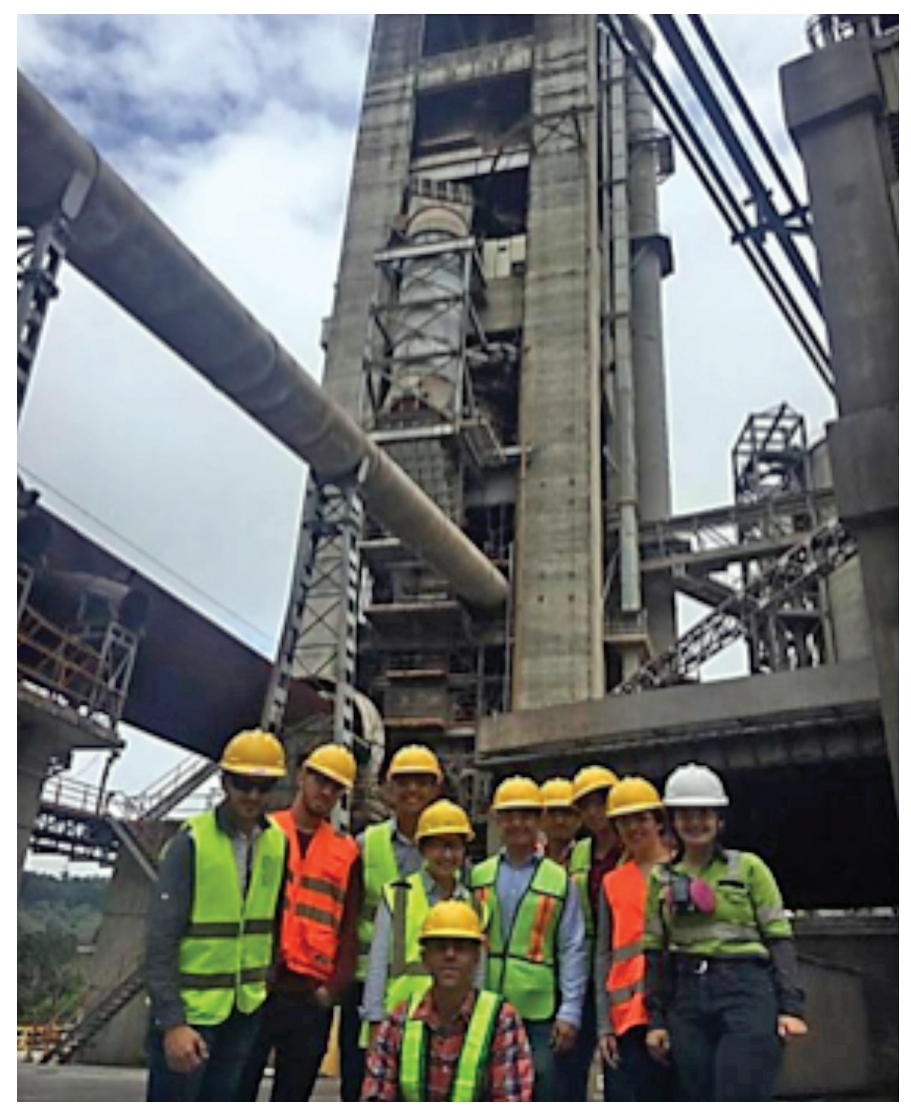

Fig.2: Visita técnica a la fábrica de cemento

Por último, pero no menos importante, cada grupo debe realizar un proyecto de investigación del algún tema en estado del arte o tecnología de punta y deben defenderlo ante un jurado integrado por profesionales externos a la Universidad que fueron los proponentes de los temas. Cada grupo es evaluado como si presentaran su trabajo final de graduación (TFG). Esto experiencia les permite recibir observaciones de mejora para que la exposición de su TFG tenga un buen nivel en contenido y forma.

\section{Testimonios}

\section{B. Marín}

Es un muy buen método puesto a que nos motiva a resolver distintos problemas investigando y preparándonos como ingenieros al punto de que concluidos los cuatro meses terminamos con un pensamiento más crítico.

También que es el único curso en el que no solo se aprende en clases sino en la calle y con problemas de la calle que es lo que uno espera puesto a que para allá vamos.

\section{W. Hernández}

En todo este tiempo me he sentido como un estudiante, esto fue sólo hasta llevar el curso de Materiales que me ha hecho sentir que estoy en una carrera de Ingeniería Civil y más allá de eso ha sido el primero en que me ha llamado Ingeniero.
Las visitas de campo y la metodología realizada me hicieron sentir que estaba desarrollándome como un ingeniero.

\section{S. Valverde}

Para mi fue un curso totalmente diferente a lo que estaba acostumbrada y llenó vacíos que tenía.

Ya concluyendo mis estudios me hubiese gustado tener más cursos con esa metodología en donde me permitieran observar y ser parte de lo que aprendo teóricamente en un salón.

\section{Bonilla}

En lo personal me cambió muchísimo la visión de lo que es recibir una clase, estudiar y aprobar un curso, a tener una conversación entre ingenieros y aprender sobre los temas relacionados con ingeniería.

Las giras ayudan a aclarar las conversaciones entre ingenieros en clase, y refuerzas las ideas y los problemas que vamos a tener cuando se ejerza profesionalmente.

\section{Conclusiones}

- El cambio inicia por los docentes. Es necesario cambiar la forma actual de enseñanza y pasar a un formato de Ingenieros experimentados que trabajen con Ingenieros en formación.

- Se aprovecha la oportunidad para ir fomentando el emprendimiento en vez de preparar empleados.

- $\quad$ Se fortalece el concepto de trabajo en equipo y de asumir responsabilidades con metas establecidas y tiempos de cumplimiento.

- Las visitas a los proyectos reafirman los conceptos, les permite desarrollar un pensamiento crítico y empiezan a desenvolverse como Ingenieros en formación.

- Los estudiantes se enfrentan a un proyecto real y retador como es la licitación y deben explotar sus virtudes y conocimiento en un ambiente competitivo.

- Las visitas técnicas cambia la rutina del campus y les permite interactuar con otros profesionales y poco a poco van elevando su nivel profesional que les permita mantener una conversación técnica con otro profesional.

- Investigar un tema novedoso les brinda la oportunidad de ver más allá de lo usual en el entorno nacional y a la vez mejoran las técnicas de presentación y comunicación de los conceptos ante un jurado profesional, llegando a ser una excelente experiencia previa a la defensa de su TFG y un insumo para exponer proyectos ante clientes. 


\section{Referencias}

[1] American Concrete Institute. (2019). ACI-211.1-91. Standard Practice for Selecting Proportions for Normal, Heavyweight, and Mass Concrete. Farmington Hills, MIUSA

[2] Portland Cement Association. (2004). Diseño y Control de Mezclas de Concreto EB201. Skokie, Illinois 60077-1083 EE. UU.: PCA.

[3] Instituto de Normas Técnicas de Costa Rica. (2018). INTE C147:2018. Cemento hidráulico. Requisitos (.). San José, Costa Rica: INTECO.

[4] Rodríguez Cruz, R (2007, enero). Compendio de estrategias bajo el enfoque por competencias, Primera versión. México: Instituto Tecnológico de Sonora.

[5] Centro Universitario Moacyr Sreder Bastos (2019). Ingeniero civil. 26 de octubre de 2019. Sitio web: https:// www.licenciaturaspregrados.com/Ingeniero-Civil/Brasil/ Centro-Universit\%C3\%A1 rio-Moacyr-Sreder-Bastos(UNIMSB)/.

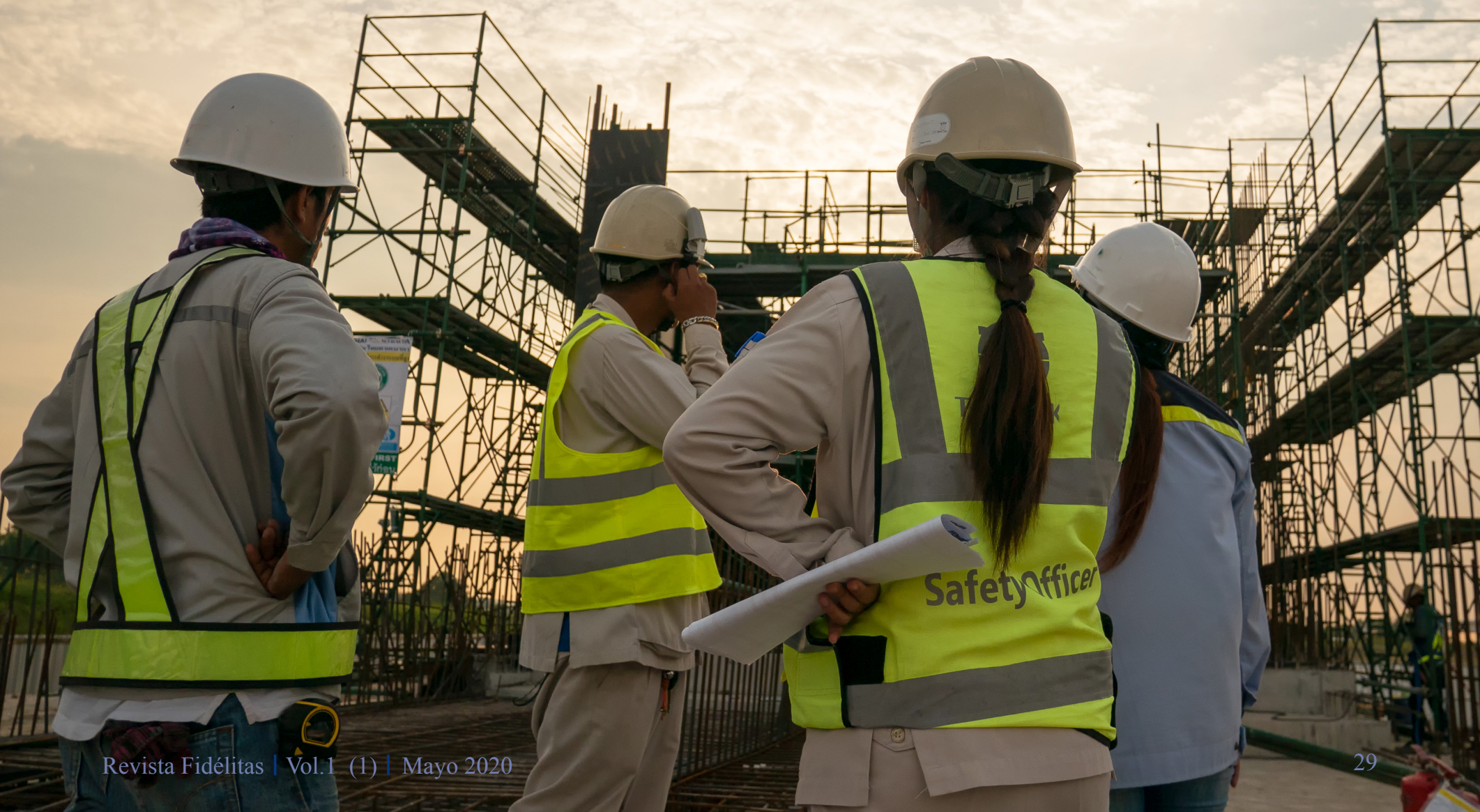

